\title{
Solubility Interactions in Compensated, Heavily Doped Germanium*
}

\author{
J. O. MCCALDIN \\ North American Aviation Science Center, Thousand Oaks, California \\ (Received 18 July 1964)
}

\begin{abstract}
The solubility of the two donors, arsenic and antimony, in Ge at $870^{\circ} \mathrm{C}$ was enhanced by the acceptor dopant $\mathrm{Ga}$ at concentrations $\geq 10^{19} \mathrm{~cm}^{-3}$. The observed enhancement is in agreement with theory of the dopant-carrier ionization equilibrium in the Boltzmann approximation and confirms the requirement of the theory that the solubility enhancement depend only on the net carrier concentration and not on the chemical identity of the donor. A contrary result would have been likely, if donor-acceptor complexes played an important role in these solubility effects. The experimentally determined intrinsic carrier concentration at $870^{\circ} \mathrm{C}$ is $n_{i}=1.8 \times 10^{19} \mathrm{~cm}^{-3}$ compared to the extrapolated Hall value of $n_{i} \sim 1.3 \times 10^{19} \mathrm{~cm}^{-3}$. The indicated extrinsic behavior implies that $p-n$ junctions persist in very heavily doped germanium to the melting point.
\end{abstract}

\section{INTRODUCTION}

$S^{\mathrm{B}}$ EVERAL years ago, Morin and Maita ${ }^{1}$ reported the intrinsic carrier concentration $n_{i}$ in germanium and silicon over a wide range of temperatures. Their measurements indicated that very heavily doped $\mathrm{Ge}$ and $\mathrm{Si}$ should remain extrinsic at elevated temperatures, indeed to the melting point. A similar conclusion applies to the III-V compounds and indeed quantitative estimates of $n_{i}$ vs temperature have recently been presented $^{2}$ for all these materials. Thus, it appears that these materials can exhibit $p$ - or $n$-type behavior during crystal growing or diffusion doping, with consequent effects on diffusivities, ion mobilities, solubilities, etc.

Such extrinsic behavior effects have been extensively studied $^{3}$ at lower temperatures in lithium-doped Ge and $\mathrm{Si}$. In this case, excellent agreement has been obtained between theory and experiment. For the case of the more usual dopants from columns III and V in Ge and $\mathrm{Si}$, however, much less is known. Extrinsic effects in diffusion have been observed by several investigators. ${ }^{4-6}$ These effects can be complicated, however, by uncertainty as to the effective charge ${ }^{7,8}$ on an atom during the diffusion process.

The present work was undertaken to establish quantitatively the extrinsic solubility interaction between a conventional substitutional donor and substitutional acceptor, whose charge states are known. In this case, the mutual solubility enhancement between the donor and acceptor can be anticipated from $n_{i}$ values obtained from Hall measurements. (A study ${ }^{9}$ of solubility in GaAs indicated a value of $n_{i}$ several times the Hall value. This result may be due to the different statistical

\footnotetext{
* The experiments described in this paper were performed while the author was at Hughes Research Laboratories.

${ }^{1}$ F. J. Morin and J. P. Maita, Phys. Rev. 94, 1525 (1954); 96, $28(1954)$

2 R. N. Hall and J. H. Racette, J. Appl. Phys. 35, 379 (1964).

${ }^{3}$ H. Reiss, C. S. Fuller, and F. J. Morin, Bell System Tech. J. 35,535 (1956).

4 J. O. McCaldin, J. Appl. Phys. 31, 89 (1960).

5 A. D. Kurtz and R. Yee, J. Appl. Phys. 31, 303 (1960)

${ }^{6} \mathrm{C}$. Fa and R. Zuleeg, Solid State Electron. 3, 18 (1961).

${ }^{7}$ C. J. Gallagher, J. Phys. Chem. Solids 3, 82 (1957).

${ }^{8}$ See, for example, Robert J. Friauf, J. Appl. Phys. 33, 494 (1962).

${ }^{9}$ J. O. McCaldin, J. Appl. Phys. 34, 1748 (1963).
}

weight assigned to light and heavy carriers by solubility and Hall measurements. In the case of germanium, however, only carriers of roughly the same mass are likely to be involved.)

\section{EXPERIMENTAL PROCEDURE AND RESULTS}

The experimental procedure (and some preliminary results) were given in detail previously ${ }^{10}$ and will only be summarized here. This procedure takes advantage of the fact that the donors, arsenic and antimony, diffuse several hundred-fold more rapidly in germanium than does the acceptor, gallium. Thus when several slices of germanium, each with a different gallium concentration, are exposed to the same vapor pressure of donor element, equilibrium can be obtained in the donor distribution, while the acceptor distribution among the various slices is affected only negligibly. In such an experiment, the vapor pressure of the donor element is a constant, the acceptor concentration is an independent experimental variable, and the donor solubility is a dependent variable. The experiment can, of course, be repeated at different vapor pressures of the donor element, as was done in the work to be described. Also the temperature during equilibration could in principle be varied; however, even near the melting point of germanium, equilibration times of $\sim 40$ days are needed. Hence the present experiments were always performed near the melting point, namely, at $870^{\circ} \mathrm{C}$.

Carrier concentrations were obtained from Hall measurements on the germanium slices before and after exposure to the vapor of the donor element. The Hall concentration before exposure indicated the gallium concentration $N_{A}$. The concentration after exposure measured the room-temperature net carrier concentration, $p-n=N_{A}-N_{D}$, where $p$ and $n$ are the hole and electron concentrations, and $N_{A}$ and $N_{D}$ are the concentrations of acceptor and donor elements. In this way the amount of donor element that goes into solution was measured as a function of the amount of acceptor present in the host crystal.

${ }^{10} \mathrm{~J}$. O. McCaldin in Metallurgy of Elemental and Compound Semiconductors, edited by Ralph O. Grubel (Interscience Publishers, Inc., New York, 1960), p. 329. 


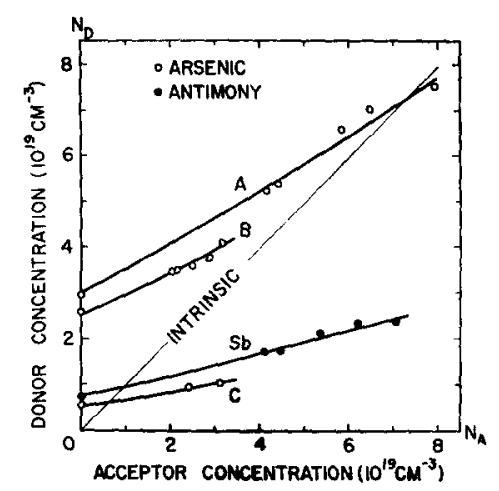

fig. 1. The solubility at $870^{\circ} \mathrm{C}$ of the donor elements, arsenic and antimony, in the presence of the acceptor element, gallium. The curves $\mathrm{A}, \mathrm{B}$, and $\mathrm{C}$ correspond to three different arsenic pressures in the system during equilibration.

The results of four such experiments are presented in Fig. 1. Clearly the donor solubility is affected by the presence of an acceptor, so that the customary assumption of dilute solution behavior is not applicable at these concentrations.

\section{DISCUSSION}

The solubility interactions demonstrated in these experiments can be compared to an approximate theory for the dopant-carrier ionization equilibrium. To make such a comparison, one needs to know the energy difference, $E_{F}-E_{D}$, where $E_{F}$ is the Fermi energy and $E_{D}$ the donor ionization energy. This energy difference specifies the degree of ionization of the donor, which in turn influences the donor solubility. To estimate this energy difference from the present data, we can use the measured net carrier concentration to locate the Fermi level with respect to the donor level.Thus the main purpose of the present discussion is to relate the enhanced donor solubility observed to net carrier concentrations.

First, we note a limitation on the accuracy with which the net carrier concentration is known in these experiments. For, unlike the chemical concentrations of dopant and acceptor, which remain the same at the elevated equilibration temperature and at room temperature, the net carrier concentration will vary somewhat with temperature. The latter concentration is fixed by the neutrality condition

$$
p-n=\left[A^{-}\right]-\left[D^{+}\right]
$$

where $p$ and $n$ are the hole and electron concentrations and $\left[A^{-}\right]$and $\left[D^{+}\right]$are the concentrations of ionized acceptors and donors, respectively. Thus the net carrier concentration is affected by de-ionization of the dopants, an effect which can be appreciable, e.g., at $870^{\circ} \mathrm{C}$ where the band gap ${ }^{11}$ may be as small as $3 k T$. In the present experiments the Fermi level is maintained within a range of $\sim 2 k T$ to minimize variations in the de-ionization effect.

The ionization of the donor centers is described by the following equation for the probability that electrons

${ }^{11}$ J. S. Blakemore, Proc. Phys. Soc. (London) 71, 692 (1958). occupy the donor levels:

$$
\frac{\left[D^{\circ}\right]}{N_{D}}=\left(1+\frac{1}{2} \exp ^{-} \frac{E_{D}-E_{F}}{k T}\right)^{-1} \sim 2 \exp \frac{E_{F}-E_{D}}{k T},
$$

where $N_{D}$ is the total concentration of donors, $\left[D^{\circ}\right]$ is the concentration of un-ionized donors, i.e., the density of electrons occupying the donor level, $k$ is Boltzmann's constant, and $T$ the absolute temperature. The exact relationship of Eq. (2) is the Fermi-Dirac expression, and is followed by the Boltzmann approximation. The factor $\frac{1}{2}$ in this expression arises from the special way of counting spin degenerate states, as described by Reiss $^{12}$ and Spenke. ${ }^{13}$ In view of the limited accuracy with which $(p-n)$ is known, as discussed above, it seems appropriate to proceed with the Boltzmann approximation. Equation (2) then indicates that in a particular experiment where the activity of the donor element is held constant, i.e., $\left[D^{\circ}\right]$ is fixed, the total donor concentration in solution $N_{D}$ varies exponentially with $E_{F}-E_{D}$.

The exponential in Eq. (2) is related to the carrier concentration by

$$
\frac{n}{N_{c}}=\frac{1}{\gamma_{n}} \exp \frac{E_{F}-E_{c}}{k T} \sim \exp \frac{E_{F}-E_{c}}{k T},
$$

where $N_{c}$ is the lumped density-of-states for the conduction band, $E_{c}$ is the energy of the conduction band edge, and $\gamma_{n}$ is an activity coefficient for electrons which has been calculated by Rosenberg ${ }^{14}$ to correct the Boltzmann factor for Fermi statistics. In the present experiments, $1<\gamma_{n} \leq 1.2$ and we again adopt the Boltzmann approximation, shown to the right in Eq. (3).

One further relation is needed to apply the present experimental data. The conduction electron concentration $n$ must be related to the measured net carrier concentration, $p-n$. From the mass-action relation: $p \times n=n_{i}^{2}$, one finds

$$
n=\left(-\frac{p-n}{2}\right)+\left[\left(\frac{p-n}{2}\right)+n_{i}^{2}\right]^{0.5}
$$

Relations (2), (3), and (4) may be combined, if one makes the usual approximation $E_{D} \simeq E_{c}$, to yield $N_{D}$ as a function of $(p-n)$.

$$
\begin{aligned}
& N_{D}=\frac{\left[D^{\circ}\right] N_{c}}{2 n_{i}^{2}} \times p \\
& =\text { const. } \times\left\{\frac{p-n}{2}+\left[\left(\frac{p-n}{2}\right)^{2}+n_{i}^{2}\right]^{0.5}\right\} .
\end{aligned}
$$

${ }^{12}$ H. Reiss, J. Chem. Phys. 21, 1209 (1953).

${ }^{13}$ Eberhard Spenke, Electronic Semiconductors (McGraw-Hill Book Company, Inc., New York, 1958), see Appendix III.

${ }^{14}$ Arthur J. Rosenberg, J. Chem. Phys. 33, 665 (1960). 
The constant in Eq. (5) remains unknown in the present experiments, but the bracketed term can be evaluated since $(p-n)$ is measured directly and $n_{i} \sim 1.3$ $\times 10^{19} \mathrm{~cm}^{-3}$ by extrapolation of Hall measurements ${ }^{1}$ at lower temperatures. Thus Eq. (5) could be used to evaluate the unknown constant $\left[D^{\circ}\right]$ and to check the four experimental runs for internal consistency.

A more direct way to check the present data against the approximate theory represented by (5) is to use the logarithmic form

$$
\log N_{D}=\text { const. }+f(p-n),
$$

which shows clearly that in a plot of $\log N_{D}$ vs $(p-n)$ all the experimental runs should exhibit the same functional dependence on $(p-n)$, differing only in an additive constant. Such a plot appears in Fig. 2. A scale of Fermi levels for $870^{\circ}$ derived from the carrier concentrations according to Eqs. (3) and (4) is included in the figure. The parameter $n_{i}$ in this plot has been chosen equal to $1.8 \times 10^{19} / \mathrm{cc}$ to give a best fit to the data. The curve fitting in this figure really amounts to drawing straight lines of the proper slope $\left(\propto n_{i}^{-1}\right)$ through the data points, since the slight curvature predicted by (5) for these plots is hardly significant in the presence of the scatter in data points. The curve fitting in this figure is significant, however, in showing how a curve of the same slope fits all four experimental runs reasonably well. A curve of the same slope applies equally well to arsenic or antimony as the donor, as required by the present theory. Incidentally, if the formation of donor-acceptor complexes ${ }^{10}$ were important in these solubility effects, one would in general not expect a
FIg. 2. The solubility of the donor elements, arsenic (open circles) and antimony (filled circles), as a function of the net carrier concentration. The curves fitting the experimental points are calculated from $\mathrm{Eq}$. (5) with $n_{i}$ $=1.8 \times 10^{19} \mathrm{~cm}^{-3}$.

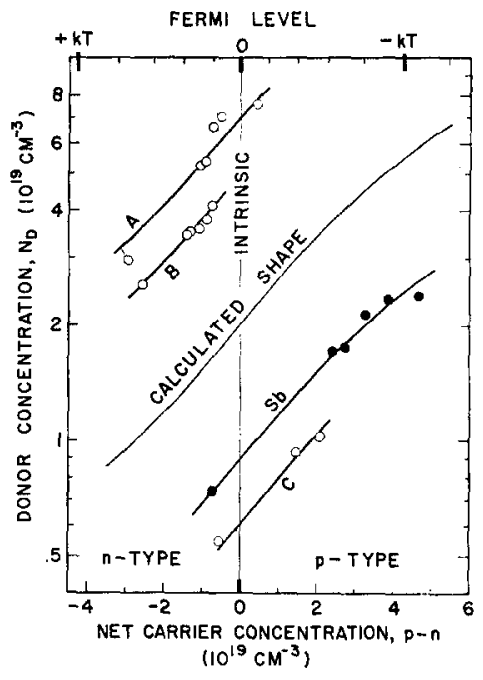

common functional dependence as shown in Fig. 2, but rather a variation from one donor to another.

The present results, in establishing certain solubility interactions, confirm that extrinsic behavior can be expected in germanium near its melting point when doping concentrations $\geq 10^{19} / \mathrm{cc}$ are used. For example, a $p n$ junction should not be "washed out" by heating heavily doped germanium to $870^{\circ} \mathrm{C}$. On the contrary, an electrostatic potential of $\sim 1.5 k T=0.15 \mathrm{eV}$ can be expected between the most $p$-type and most $n$-type doping of the present investigation. Such potentials can be expected to affect not only solubility and related thermodynamic properties, but also the dopant diffusivities. 